\title{
Ducto-lobular carcinoma in situ: Clinical and pathological findings in three cases with review of literature
}

\author{
J aspreet Ahuja ${ }^{1}$, Louise Bordeleau ${ }^{1,2}$, Anita Bane ${ }^{1,2,3}$ \\ 1. Department of Oncology, McMaster University, Hamilton, ON, Canada. 2. Juravinski Cancer Center, Hamilton, ON, \\ Canada. 3. Department of Pathology \& Molecular Medicine, McMaster University, Hamilton, ON, Canada.
}

Correspondence: Anita Bane, MB, BCh, FRCPath, PhD. Address: Department of Pathology, J uravinski Cancer Center, 699 Concession Street, Hamilton, ON, L8V 5C2, Canada. E-mail: bane@hhsc.ca

Received: September 14, 2014

Accepted: April 6, 2015

Online Published: May 11, 2015

DOI : $10.5430 / c r c p . v 2 n 3 p 72$

URL: http://dx.doi.org/10.5430/crcp.v2n3p72

\begin{abstract}
In situ carcinomas of the breast are traditionally divided into ductal and lobular based on a combination of morphological features. Ductal carcinoma in situ (DCIS) is further differentiated from lobular carcinoma in situ (LCIS) by the presence of membranous E-cadherin protein expression. The expression of this protein is lacking in the neoplastic cells of LCIS due to underlying molecular alterations in the E-cadherin gene (CDH1) but present in cases of DCIS. Pathologists frequently employ the use of E-cadherin immunohistochemistry as an ancillary aid to distinguish DCIS from LCIS in morphologically ambiguous cases. In a small minority of in situ carcinomas a dual population of neoplastic cells is present within the same terminal duct lobular unit with morphological and/or immunohistochmeical evidence of both ductal and lobular histology, an entity we and others refer to as ducto-lobular carcinoma in situ (DLCIS). Herein we describe three such cases in detail to include morphological features, E-cadherin immunohistochemical staining pattern, clinical history, management and follow-up. In addition a comprehensive review of the literature of such cases is summarised and an algorithmic approach to their future diagnosis proposed.
\end{abstract}

\section{Key words}

Ductal carcinoma in situ, Lobular carcinoma in situ, Ducto-lobular carcinoma in situ, E-cadherin

\section{I ntroduction}

In situ carcinomas of the breast are traditionally classified as ductal or lobular based on a combination of distinguishing morphological and cytological features ${ }^{[1,2]}$. This distinction is clinically important as ductal carcinoma in situ (DCIS) is treated as a de facto malignancy with complete surgical excision, radiation and for some, endocrine therapy, whereas lobular carcinoma in situ (LCIS) is considered a non-obligate precursor lesion and is followed clinically and radiologically with screening mammography (MRI is currently debated and not recommended for all patients). Chemoprevention with endocrine therapies is also an option. LCIS is further distinguished from DCIS by molecular means. LCIS is characterised by alterations in the E-cadherin-catenin complex resulting in the loss of E-cadherin, $\beta$-catenin and 120 protein expression at the cellular membrane, whereas in DCIS this complex is intact ${ }^{[3]}$. This fact has been exploited by surgical pathologists 
who utilise E-cadherin immunohistochemistry (IHC) in association with morphological features to distinguish cases of DCIS (E-cadherin positive) from LCIS (E-cadherin negative) in diagnostic pathology ${ }^{[4-7]}$.

An unforeseen consequence of this wide spread adoption of E-cadherin immunohistochemistry into diagnostic breast pathology has been the identification and acceptance of histological variants of LCIS, to include pleomorphic LCIS and LCIS with central necrosis ${ }^{[6,8,9]}$. Prior to the utilization of E-cadherin IHC these variants were commonly misdiagnosed as DCIS or termed ducto-lobular carcinoma in situ (DLCIS) or carcinoma with indeterminate features, because of their ambiguous histological characteristics. However, we and others believe that the term DLCIS is now best reserved for those lesions that truly display a mixed cell population, both ductal and lobular, within the same terminal duct lobular unit ${ }^{[10,11]}$. This dual cell population should be further confirmed with the aid of E-cadherin $\mathrm{IHC}^{[5,6]}$. Using these refined diagnostic criteria very few true cases of DLCIS have been reported as such in the literature. Herein, we report three cases of DLCIS and describe in detail their clinical presentation, pathologic appearance and pattern of expression of proteins of the E-cadherin-catenin complex. Furthermore, we propose an algorithmic approach to their diagnosis.

\section{Methods}

Cases were retrieved from the diagnostic surgical pathology files of the Juravinski Hospital and Cancer Centre and personal consult files of the study pathologist (AB) for the period 2008-2010. Three cases were found and re-reviewed. Hematoxylin and eosin stained slides were available on all three cases and limited additional tissue was available for immunohistochemical studies. Four-micron sections were cut and stained for E-cadherin (clone NCH-38 from DAKO at 1/100 dilution). Sections were developed with diaminobenzidine tetrahydrochloride and counterstained in Mayer's hematoxylin.

E-cadherin staining was considered positive when the neoplastic population of cells of interest showed a strong circumferential cell membrane staining. Absent cell membranous staining was considered negative. Positive and negative controls were included in each staining run. In all cases normal breast epithelium was present on each slide and served as an internal positive control for E-cadherin staining.

The study was approved by the Research Ethics Board (Hamilton Health Sciences, Hamilton, ON).

\section{Results}

Clinical \& Histopathological Data (see Table 1):

Table 1. Clinical and pathological summary of cases

\begin{tabular}{|c|c|c|c|c|c|c|c|c|}
\hline Case & Age/Gender & Mammography & $\begin{array}{l}\text { Co-existing } \\
\text { pathology }\end{array}$ & Grade & E-Cadherin & Surgery & Radiation & $\begin{array}{l}\text { Systemic } \\
\text { therapy }\end{array}$ \\
\hline 1 & $73 / \mathrm{F}$ & Micro-calcifications & DCIS & Low & Heterogenous & Lumpectomy & Yes & No \\
\hline 2 & $67 / F$ & Micro-calcifications & $\begin{array}{l}\text { DCIS } \\
\text { IDC (NOS) } \\
\text { ER/PR (-) } \\
\text { HER-2 (+) }\end{array}$ & Low & Heterogenous & $\begin{array}{l}\text { Mastectomy } \\
\text { SLND }\end{array}$ & No & No \\
\hline 3 & $39 / F$ & Micro-calcifications & DCIS $^{*}$ & Low & Heterogenous & $\begin{array}{l}\text { Bilateral } \\
\text { mastectomy } \\
\text { and left } \\
\text { SLND }\end{array}$ & No & Tamoxifen \\
\hline
\end{tabular}

Note. F (female); DCIS (ductal carcinoma in situ); IDC (invasive ductal carcinoma); NOS (not otherwise specified); ER (estrogen receptor); PR (progesterone receptor); SLND (sentinel lymph node dissection); ${ }^{*}$ Contralateral mastectomy had multi-centric DCIS with a single focus of microinvasive breast cancer and no lymph node involvement. 


\subsection{Case 1}

The first patient was a 73-year-old female with suspicious micro-calcifications noted on screening mammography. Her past medical history was significant for idiopathic thrombocytopenia purpura (ITP), hypertension and osteopenia. She has no significant risk factors for breast cancer. She was treated with wire localization and excision of the area of calcification. The pathology revealed fibrocystic change, proliferative disease without atypia together with a TDLU expanded by a proliferation of low-grade neoplastic cells (see Figure 1A). In some areas of the expanded TDLU the neoplastic cells had a complex architectural growth pattern to include cribiform formation whereas in other areas they displayed a solid growth pattern with cellular discohesion and scattered intracytoplasmic lumina. Calcifications were seen in association with the neoplastic epithelium and within the (benign) stoma. E-cadherin staining of the TDLU in question, showed strong complete membranous positivity in the cells forming the cribiform structures whereas the adjacent cells displaying a solid growth pattern with cellular discohesion demonstrated a complete absence of staining for E-cadherin, confirming the dual cell population (see Figure 1B). The lesion measured 5mm in maximum extent. A diagnosis of low-grade ducto-lobular carcinoma in situ was made and the patient was treated with adjuvant breast radiation therapy.
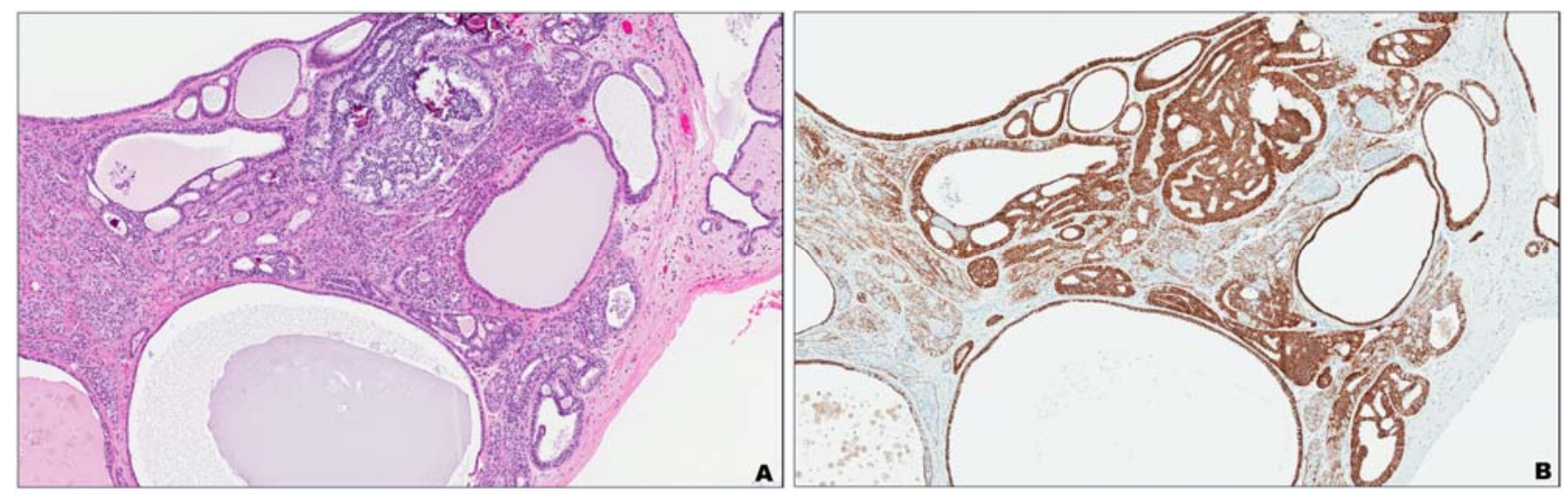

Figure 1. A: Case 1 illustrating an admixture of LCIS predominantly on the left on the picture and low grade DCIS on the right, within the same TDLU (H\&E stained, 10× magnifications). B: Case 1 stained with E-cadherin illustrating the membranous staining of the DCIS and the absence of staining in the areas of LCIS.

\subsection{Case 2}

The second patient was a 67 year old female who was diagnosed with DCIS on core biopsy following the identification of micro-calcifications on routine mammographic screening. Her past medical history included hypercholesterolemia, hypertension, depression, mitral valve prolapsed, spondylitis, and multiple myeloma. She had a significant family history of breast cancer. Genetic testing did not identify a pathogenic mutation in either the BRCA1 or BRCA2 gene.

She was treated with a left modified radical mastectomy and sentinel lymph node sampling. No mass lesion was observable on macroscopic examination and the specimen was x-rayed and areas of specimen calcification were correlated with a pre-operative digital mammogram and extensively sampled. An $8 \mathrm{~mm}$ focus of IDC (NOS), Grade II, was identified microscopically with an extensive intraductal component. The invasive cancer was ER and PR negative and HER-2 positive. There was no evidence of lymphovascular space invasion or lymph node involvement. The intraductal (ductal carcinoma in situ) component was nuclear grade III with comedo necrosis and extended over an area of $2.3 \mathrm{~cm}$. In addition there was a low-grade neoplastic population of cells present in ducts and lobules lateral to and separate from the main tumour mass. This neoplastic population of cells was morphologicaly dissimilar to the main tumour described above and had morphological and cytological features suggestive of both a low grade ductal and lobular neoplastic process including; a monotonous population of cells, cellular discohesion, intracytoplasmic lumina and focal secondary lumen formation (see Figure 2A). Immunohistochemistry for E-cadherin showed that some cells retained a crisp membranous positivity whereas others within the same TDLU had absent staining, confirming the dual cell population suspected on H\&E examination (see Figure 2B). 


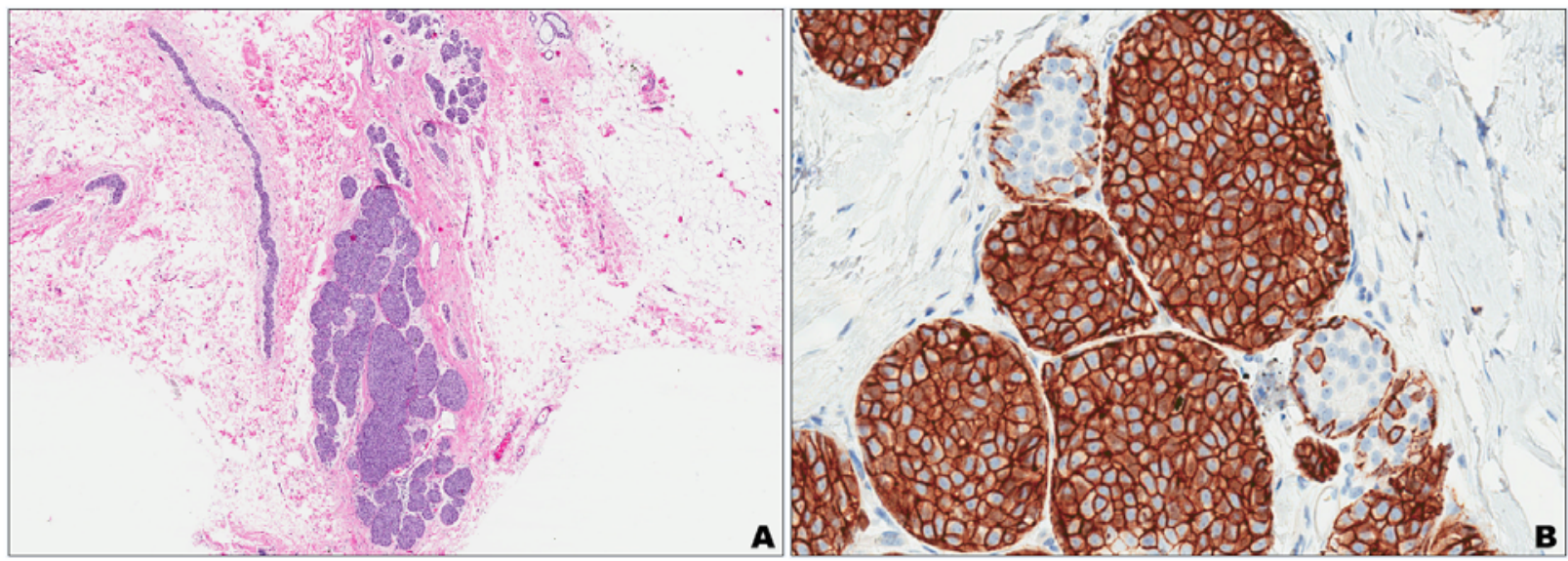

Figure 2. A: Case 2, illustrating a solid proliferation of low-grade neoplastic cells in a TDLU (H\&E stained, 5× magnifications). B: Case 2 stained with E-cadherin, illustrating the admixture of positive and negatively stained neoplastic cells within the same TDLU.

The patient did not receive adjuvant radiation therapy as she underwent a mastectomy. Endocrine treatment was not administered as the invasive tumor was hormone receptor negative and a decision to withhold adjuvant chemotherapy and herceptin was made following careful consideration and discussion. Clinical follow-up and radiologic screening of the contralateral breast continue.

\subsection{Case 3}

The third patient was a 39 year old woman who underwent a bilateral mastectomy. She had a personal history of Hodgkin's lymphoma as an adolescent and had received mantle irrradition, consequently she was undergoing annual screening digital mammography. She has a significant family history of breast cancer. Genetic testing did not identify a pathogenic mutation in either the BRCA1 or BRCA2 gene. Screening mammography revealed an area of suspicious micro-calcifications and DCIS was diagnosed on needle core biopsy on the left breast. The patient elected to have an ipsilateral therapeutic mastectomy with sentinel lymph node dissection and a contralateral prophylactic mastectomy. Multi-centric DCIS (grade III with comedo necrosis) was diagnosed in the left breast with a single focus of microinvasion with no lymph node involvement. ER and PR could not be completed on the invasive cancer as the microscopic focus of invasion was not present on deeper sections submitted for IHC, but both ER and PR were strongly positive in the adjacent DCIS. HER-2 was negative. No gross lesion was evident on macroscopic examination of the right breast but random sections revealed fibrocystic change, proliferative disease without atypia and a $4 \mathrm{~mm}$ focus of a low grade in situ process with features indeterminate for LCIS and DCIS (see Figure 3A). E-cadherin immunohistochemistry revealed that the lesion in question showed a variable staining pattern with some cells retaining a strong membranous positivity and others showing complete loss of staining (see Figure 3B). The patient received adjuvant tamoxifen.

\section{Discussion}

The pathologic entity of DLCIS encompasses two types of lesions described in this short case series. The first, illustrated by case 1 , is characterised by the intimate admixture of two separate populations of low grade neoplastic cells, individually recognisable as ductal and lobular by classic morphologic criteria, within the same terminal duct lobular unit (TDLU). The second, illustrated by cases 2 and 3 comprises a low-grade neoplastic population of cells with morphologic features indeterminate for either ductal or lobular cells present within the same TDLU. Immunohistochemical staining for E-cadherin confirmed the dual population of cells in both types of lesions, with some cells illustrating a crisp circumferential membranous positivity for E-cadherin and other cells showing absent membranous staining for this protein. 

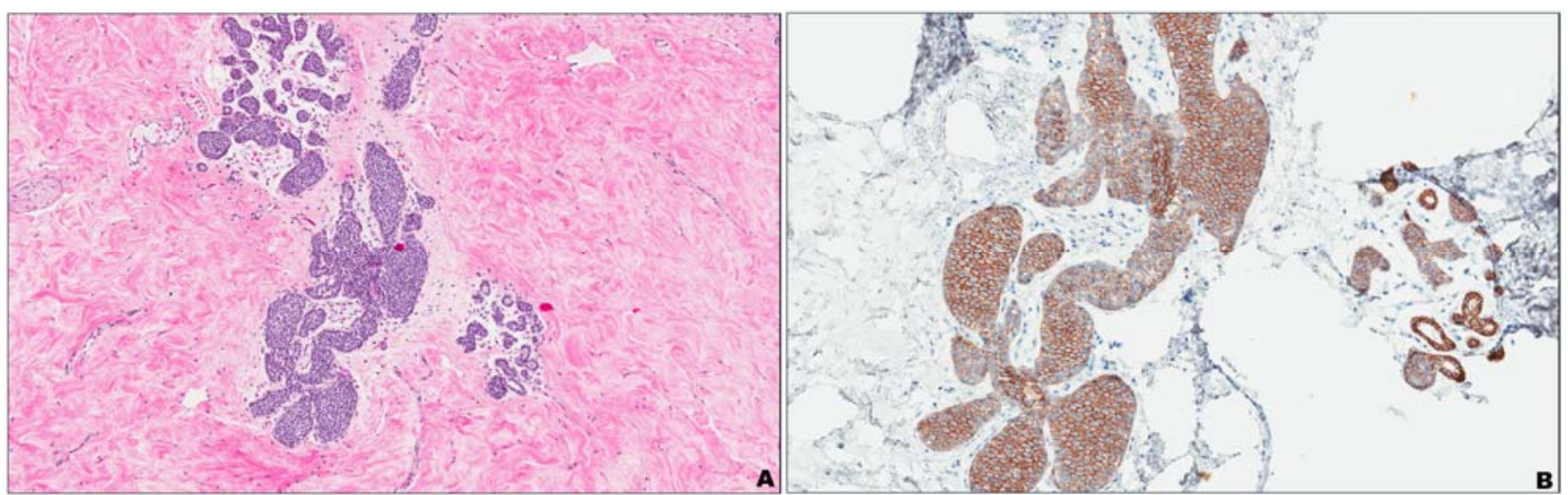

Figure 3. A: Case 3, a solid proliferation of low-grade neoplastic cells in a TDLU with morphological features indeterminate for either DCIS or LCIS (H\&E stained, $5 \times$ magnifications). B: Case 3 stained with E-cadherin illustrating positive and negatively stained neoplastic cells within the same TDLU (40× magnification).

E-cadherin is a transmembrane protein expressed in normal luminal epithelial cells of the breast and functions in cell-cell adhesion ${ }^{[12]}$. The intracellular domain of this protein complexes with $\alpha, \beta$, and p120 catenins, which connect the adhesion complex to the actin cytoskeleton ${ }^{[12]}$. Expression of E-cadherin has been shown to be lost in LCIS but not in DCIS ${ }^{[3,6,13]}$. This loss of E-cadherin is mirrored by the loss of $\beta$-catenin, $\alpha$-catenin staining and by the aberrant cytoplasmic localisation of p120 catenin in lobular neoplasia but not in DCIS ${ }^{[3,14]}$. This distinguishing immunohistochemical profile has been utilised by pathologists to reliably distinguish between DCIS and LCIS particularly in morphologically ambiguous cases. Indeed the use of ancillary immunohistochemistry in these cases led to the acceptance of newer entities such as pleomorphic lobular carcinoma in situ (PLCIS) and LCIS with central necrosis as being truly lobular lesions despite the high nuclear grade and central necrosis respectively that characterise these lesions ${ }^{[6,8,9,15]}$. When these now well accepted variants of lobular neoplasia are removed from the equation there are still a small number of cases that defy accurate classification as ductal or lobular on morphology alone and where the use of IHC for E-cadherin demonstrates two populations of neoplastic cells; those with strong circumferential membranous E-cadherin staining and those devoid of such a pattern of staining. It is this entity that we propose the term DLCIS be reserved for (see Figure 4).

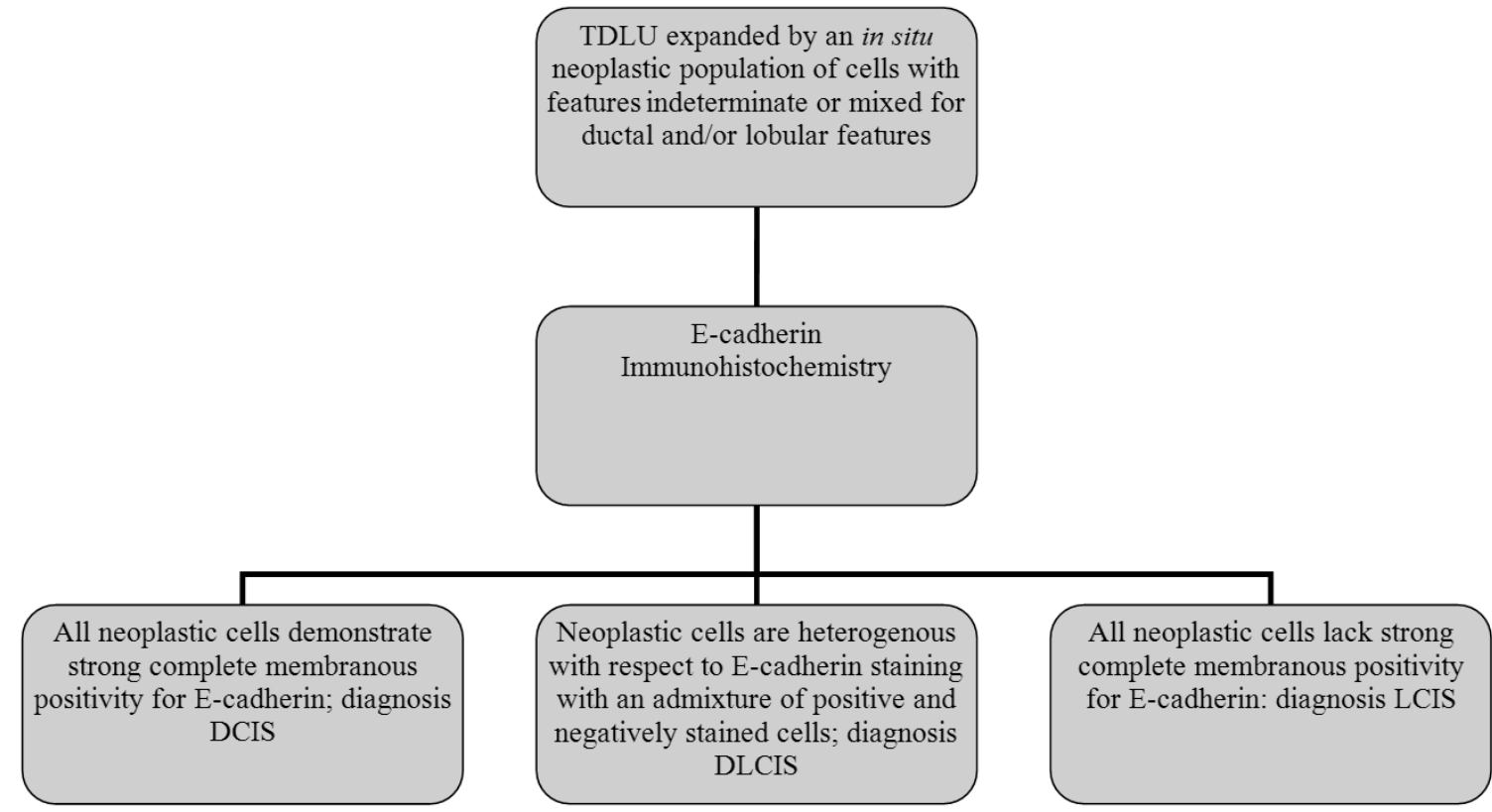

Figure 4. An algorithmic approach to the diagnosis of DLCIS using a combination of morphological and immunohistochemical characteristics 
Ductal and lobular carcinomas are both believed to arise from malignant transformation of luminal epithelial cells of the TDLU and indeed more recent molecular studies have provided evidence that lobular neoplasia and low-grade DCIS often co-exist in the same breast and show a similar profile of genetic alterations to include a diploid or near diploid karyotype, 16q loss and 1q gain ${ }^{[16]}$. Furthermore, a similar spectrum of genetic alterations have also been identified in invasive lobular carcinomas and invasive low-grade ductal carcinomas and some special type tumours to include tubular and tubulo-lobular carcinomas suggesting that a family of low-grade neoplasias exist in the breast to include LCIS, low-grade DCIS, ILC and low grade IDC which may arise from a common progenitor cell or a progenitor cell that has accumulated a similar array of genetic changes ${ }^{[16]}$. Thus it is perhaps not surprising that lobular and low-grade ductal carcinoma cells can be identified at an early precursor stage within the same TDLU as we describe herein. The small size of these lesions precluded us from performing a genetic analysis of the constituent cells.

This entity has been recognised by a number of authors under a number of names in the past and Table 2 summarises those findings. In brief the majority of cases described were low grade in nature, and occurred in females ranging in age from 42-66 years of age, E-cadherin IHC was used in all cases with the exception of Rosens case series to determine a diagnosis. The cases described include examples where LCIS and DCIS co-exits within the same TDLU ${ }^{[5,17,18]}$ (as in our case 1) or where the morphology of the neoplastic cell population was ambiguous or indeterminate and immunohistochemical staining for E-cadherin was heterogeneous with a mixed population of E-cadherin negative and E-cadherin positive cells visible $^{[6,7,11,17,19,20]}$. Twenty-three of the 64 cases described in the literature had significant co-existent pathology including invasive carcinoma, DCIS or LCIS. In the remainder of the described cases DLCIS appears to have been the index lesion, for these cases treatment and follow-up are not discussed.

Table 2. Summary of DLCIS cases reported

\begin{tabular}{|c|c|c|c|c|c|c|}
\hline Author & $\begin{array}{l}\text { Number } \\
\text { of cases }\end{array}$ & Grade of lesion & Age & Additional Pathology & Treatment & Outcome \\
\hline $\begin{array}{l}\text { Rosen PP } \\
{[17]}\end{array}$ & 3 & Not specified & $58,46,42$ & $\begin{array}{l}2 \text { patients had } \\
\text { co-existant ipsilateral } \\
\text { IDC }\end{array}$ & Mastectomy & $\begin{array}{l}\text { Alive and well at } \\
12 \text { years and } 13 \\
\text { months, no } \\
\text { follow-up reported } \\
\text { on third case }\end{array}$ \\
\hline $\begin{array}{l}\text { Coyne JD } \\
{[18]}\end{array}$ & 1 & $\begin{array}{l}\text { Low, intermediate } \\
\text { and high grade DCIS }\end{array}$ & 45 & $\begin{array}{l}\text { Co-existant DCIS and } \\
\text { LCIS }\end{array}$ & Mastectomy & $\begin{array}{l}\text { Alive and well } \\
\text { after } 5 \text { years }\end{array}$ \\
\hline $\begin{array}{l}\text { Bratthauer } \\
\text { GL }^{[20]}\end{array}$ & 27 & Not specified & Not specified & Index lesion & Not specified & Not specified \\
\hline $\begin{array}{l}\text { Fisher ER } \\
\text { [10] }\end{array}$ & 6 & Not specified & Not specified & Index lesion & Local excision & Not specified \\
\hline $\begin{array}{l}\text { Jacobs TW } \\
\text { [13] }\end{array}$ & 6 & Low Grade & Not specified & Index lesion & Not specified & Not specified \\
\hline $\operatorname{Acs} G^{[5]^{*}}$ & 3 & Not specified & Not specified & Not specified & Not specified & Not specified \\
\hline $\begin{array}{l}\text { Goldstein } \\
\mathrm{NS}^{[19]}\end{array}$ & 17 & Low Grade & Not specified & Not specified & Not specified & Not specified \\
\hline $\begin{array}{l}\text { Maluf HM } \\
\text { [7] }\end{array}$ & 2 & Low Grade & 66,65 & $\begin{array}{l}\text { Both patients had } \\
\text { ipsilateral IDC and ILC }\end{array}$ & Not specified & Not specified \\
\hline
\end{tabular}

Note. ${ }^{*}$ The authors discuss 14 cases of what they describe as DLCIS however under these are examples of PLCIS, distinct from these cases they mention briefly 3 in situ lesions with concurrent DCIS and LCIS in the same TDLU. The dual cell population is confirmed by E-cadherin immunohistochemistry. DLCIS: Ducto-lobular carcinoma in situ; DCIS: Ductal carcinoma in situ; LCIS: Lobular carcinoma in situ; IDC: Invasive ductal carcinoma; ILC: Invasive lobular carcinoma

The appropriate treatment for patients with DLCIS has not been established although some authors have recommended that they should be treated as DCIS rather than LCIS because of the putative ductal origin ${ }^{[6,11]}$. Current guidelines for DCIS include complete surgical excision, followed by breast irradiation in the case of breast conserving surgery and adjuvant tamoxifen where appropriate. In our series the one patient in whom the diagnosis was made on a wire localisation 
biopsy specimen and in whom DLCIS was the most significant pathological finding, went on to receive local breast irradiation. Both of the remaining two patients underwent mastectomy, one received adjuvant tamoxifen and the other did not. All patients are currently alive without recurrence.

In summary, DLCIS is characterised by the presence of low-grade neoplastic cells with ductal and lobular features both morphologically and immunohistochemically in the same TDLU. We propose an algorithmic approach to the diagnosis of such lesions. Due to the relative rarity of such lesions, the lack of well described treatment approaches and accurate prognostic information a coherent approach to their recognition and reporting is clinically important.

\section{References}

[1] O'Malley FP, Pinder SE. Breast Pathology (Foundations in Diagnostic Pathology). Churchill Livingstone. 2011.

[2] Page DL, Anderson TJ. Diagnostic Histopathology of the Breast. Churchill Livingstone, Edinburgh, Scotland, 1987.

[3] Mastracci TL, Tjan S, Bane AL, et al. E-cadherin alterations in atypical lobular hyperplasia and lobular carcinoma in situ of the breast. Mod Pathol. 2005; 18: 741-751. PMid: 15696125. http://dx.doi.org/10.1038/modpathol.3800362

[4] Vos CB, Cleton-Jansen AM, Berx G, et al. E-cadherin inactivation in lobular carcinoma in situ of the breast: an early event in tumorigenesis. Br J Cancer. 1997; 76: 1131-1133. PMid: 9365159. http://dx.doi.org/10.1038/bjc.1997.523

[5] Acs G, Lawton TJ, Rebbeck TR, et al. Differential expression of E-cadherin in lobular and ductal neoplasms of the breast and its biologic and diagnostic implications. Am J Clin Pathol. 2001; 115: 85-98. PMid: 11190811. http://dx.doi.org/10.1309/FDHX-L92R-BATQ-2GE0

[6] Jacobs TW, Pliss N, Kouria G, et al. Carcinomas in situ of the breast with indeterminate features: role of E-cadherin staining in categorization. Am J Surg Pathol. 2001; 25: 229-236. PMid: 11176072. http://dx.doi.org/10.1097/00000478-200102000-00011

[7] Maluf HM, Swanson PE, Koerner FC. Solid low-grade in situ carcinoma of the breast: role of associated lesions and E-cadherin in differential diagnosis. Am J Surg Pathol. 2001; 25: 237-244. PMid: 11176073. http://dx.doi.org/10.1097/00000478-200102000-00012

[8] Fadare O, Dadmanesh F, Alvarado-Cabrero I, et al. Lobular intraepithelial neoplasia [lobular carcinoma in situ] with comedo-type necrosis: A clinicopathologic study of 18 cases. Am J Surg Pathol. 2006; 30: 1445-1453. PMid: 17063087. http://dx.doi.org/10.1097/01.pas.0000213290.58283.82

[9] Sneige N, Wang J, Baker BA, et al. Clinical, histopathologic, and biologic features of pleomorphic lobular (ductal-lobular) carcinoma in situ of the breast: a report of 24 cases. Mod Pathol. 2002; 15: 1044-1050. PMid: 12379750. http://dx.doi.org/10.1097/01.MP.0000030450.20581.E3

[10] Fisher ER, Costantino J, Fisher B, et al. Pathologic findings from the National Surgical Adjuvant Breast Project (NSABP) Protocol B-17. Five-year observations concerning lobular carcinoma in situ. Cancer. 1996; 78: 1403-1416. http://dx.doi.org/10.1002/(SICI)1097-0142(19961001)78:7<1403::AID-CNCR6>3.0.CO;2-L

[11] Fisher ER, Land SR, Fisher B, et al. Pathologic findings from the National Surgical Adjuvant Breast and Bowel Project: twelve-year observations concerning lobular carcinoma in situ. Cancer. 2004; 100: 238-244. PMid: 14716756. http://dx.doi.org/10.1002/cncr.11883

[12] van Roy F, Berx G. The cell-cell adhesion molecule E-cadherin. Cell Mol Life Sci. 2008; 65: 3756-3788. PMid: 18726070. http://dx.doi.org/10.1007/s00018-008-8281-1

[13] Jacobs TW. Atypical Lobular Hyperplasia (ALH) and Lobular Carcinoma in situ (LCIS) Including 'Pleomorphic Variant'. In: Anonymous, 1st edn. Churchill Livingstone: Elsevier; 2006.

[14] Dabbs DJ, Bhargava R, Chivukula M. Lobular versus ductal breast neoplasms: the diagnostic utility of p120 catenin. Am J Surg Pathol. 2007; 31: 427-437. PMid: 17325485. http://dx.doi.org/10.1097/01.pas.0000213386.63160.3f

[15] Chivukula M, Haynik DM, Brufsky A, et al. Pleomorphic lobular carcinoma in situ (PLCIS) on breast core needle biopsies: clinical significance and immunoprofile. Am J Surg Pathol. 2008; 32: 1721-1726. PMid: 18769331. http://dx.doi.org/10.1097/PAS.0b013e31817dc3a6

[16] Abdel-Fatah TM, Powe DG, Hodi Z, et al. Morphologic and molecular evolutionary pathways of low nuclear grade invasive breast cancers and their putative precursor lesions: further evidence to support the concept of low nuclear grade breast neoplasia family. Am J Surg Pathol. 2008; 32: 513-523. PMid: 18223478. http://dx.doi.org/10.1097/PAS.0b013e318161d1a5

[17] Rosen PP. Coexistent lobular carcinoma in situ and intraductal carcinoma in a single lobular-duct unit. Am J Surg Pathol. 1980; 4 : 241-246. PMid: 6249133. http://dx.doi.org/10.1097/00000478-198006000-00004 
[18] Coyne JD, Dervan PA, Barr L, et al. Mixed apocrine/endocrine ductal carcinoma in situ of the breast coexistent with lobular carcinoma in situ. J Clin Pathol. 2001; 54: 70-73. PMid: 11271793. http://dx.doi.org/10.1136/jcp.54.1.70

[19] Goldstein NS, Kestin LL, Vicini FA. Clinicopathologic implications of E-cadherin reactivity in patients with lobular carcinoma in situ of the breast. Cancer. 2001; 92: 738-747.

http://dx.doi.org/10.1002/1097-0142(20010815)92:4<738::AID-CNCR1377>3.0.CO;2-Z

[20] Bratthauer GL, Moinfar F, Stamatakos MD, et al. Combined E-cadherin and high molecular weight cytokeratin immunoprofile differentiates lobular, ductal, and hybrid mammary intraepithelial neoplasias. Hum Pathol. 2002; 33: 620-627. PMid: 12152161. http://dx.doi.org/10.1053/hupa.2002.124789 